\title{
Uncertainties in the Magnetic Field of the Milky Way
}

\author{
Michael Unger* \\ Institute for Nuclear Physics, Karlsruhe Institute of Technology, 76344 \\ Eggenstein-Leopoldshafen, Germany \\ E-mail: michael.unger@kit.edu
}

\section{Glennys R. Farrar}

Center for Cosmology and Particle Physics, New York University, New York, NY 10003, USA

E-mail: gf25@nyu.edu

\begin{abstract}
We improve on the model of the Galactic Magnetic Field (GMF) from Jansson \& Farrar (2012), which was constrained using all-sky rotation measures of extragalactic sources and polarized and unpolarized synchrotron emission data from WMAP. We have

- developed several alternative functional forms for the coherent and random components

- used newer synchrotron products from Planck and WMAP

- used new models of the densities of thermal electrons and cosmic-ray electrons.

The differences in the resultant GMF models, depending on which parameterization of the field, synchrotron product and electron densities are used, provides a measure of the uncertainty in our inference of the GMF. We discuss the impact of these uncertainties on charged-particle astronomy at ultra-high energies.
\end{abstract}

35th International Cosmic Ray Conference - ICRC2017

10-20 July, 2017

Bexco, Busan, Korea

${ }^{*}$ Speaker. 


\section{Introduction}

Spiral galaxies such as the Milky Way are known to be permeated by magnetic fields (see e.g. [1, 2, 3]). Global models of these fields are frequently used to study a variety of astrophysical phenomena including star formation, gas dynamics, the propagation and acceleration of low-energy cosmic rays and the deflections of the arrival directions of ultrahigh-energy cosmic rays.

The hitherto most complete attempt to determine the global structure of the Galactic magnetic field (GMF) is the model of Jansson \& Farrar [4, 5] (JF12). In this model, the GMF is described by a superposition of three divergence-free large-scale regular components: a spiral disk field, a toroidal halo field and a poloidal field ("X-field"). In addition, there is a turbulent field model whose disk component is modeled following the same spiral structure as the regular component and there is also an extended random halo field. The $22+14$ parameters of the regular and random magnetic field are constrained by:

(a) Multi-frequency radio observations of the Faraday rotation of extragalactic radio sources. The corresponding rotation measures (RMs) are proportional to the line-of-sight integral of the longitudinal magnetic field, weighted with the density of thermal electrons of the warm ionized medium of the Galaxy.

(b) Measurements of the polarized synchrotron emission of cosmic-ray electrons in the regular magnetic field of the Galaxy. The total polarized intensity (PI) is proportional to the line of sight integral of the ordered component of the transverse magnetic field strength, weighted by the density of cosmic-ray electrons. The direction of the transverse magnetic field component can be inferred from the Stokes parameters Q and U.

(c) Measurements of the total (polarized and unpolarized) synchrotron intensity I, which is a line-of-sight integral depending on the product of cosmic-ray electron density and total transverse magnetic field strength (coherent and random).

In this contribution we investigate the uncertainties in our inference of the GMF introduced by the various assumptions entailed in the global modeling of the field, especially the functional forms used for the field characterizations and the models for the thermal and cosmic-ray electrons. In addition, we consider uncertainties due to uncertainties in the observables used to constrain the field model.

After describing the data analysis procedure in the next section, various model variations will be discussed in Sec. 3. The finite number of variations investigated can of course not provide an exhaustive investigation of models compatible with the data, therefore the results presented in this article provide a lower limit on the uncertainties of the GMF, in the absence of further input to select among or discard some of the GMF model variations. As an example of the impact of the uncertainties, we discuss in Sec. 4 the uncertainties of the arrival directions of ultrahigh energy cosmic rays due to the deflections in an uncertain Galactic magnetic field.

The analysis presented here follows closely the procedure developed for the JF12 model. We use the same catalogue of 40403 extragalactic RMs and bin it into 3072 equal-area HEALPix pixels on the sky to define the mean and variance of the RMs in a particular direction. A similar procedure is applied to the synchrotron maps from WMAP and Planck. For each model of the GMF we produce predictions of the observables RM, Q, U and I by a line-of-sight integration of 
the magnetic field weighted with the thermal electrons density and cosmic-ray electrons spectrum respectively. For this purpose we developed a new computational framework that allows for adaptive step control during the integration and a highly modular model configuration that is needed for the variations described in the next section. A comparison of the measured observables with the simulated predictions from the JF12 model is shown in the figure below.
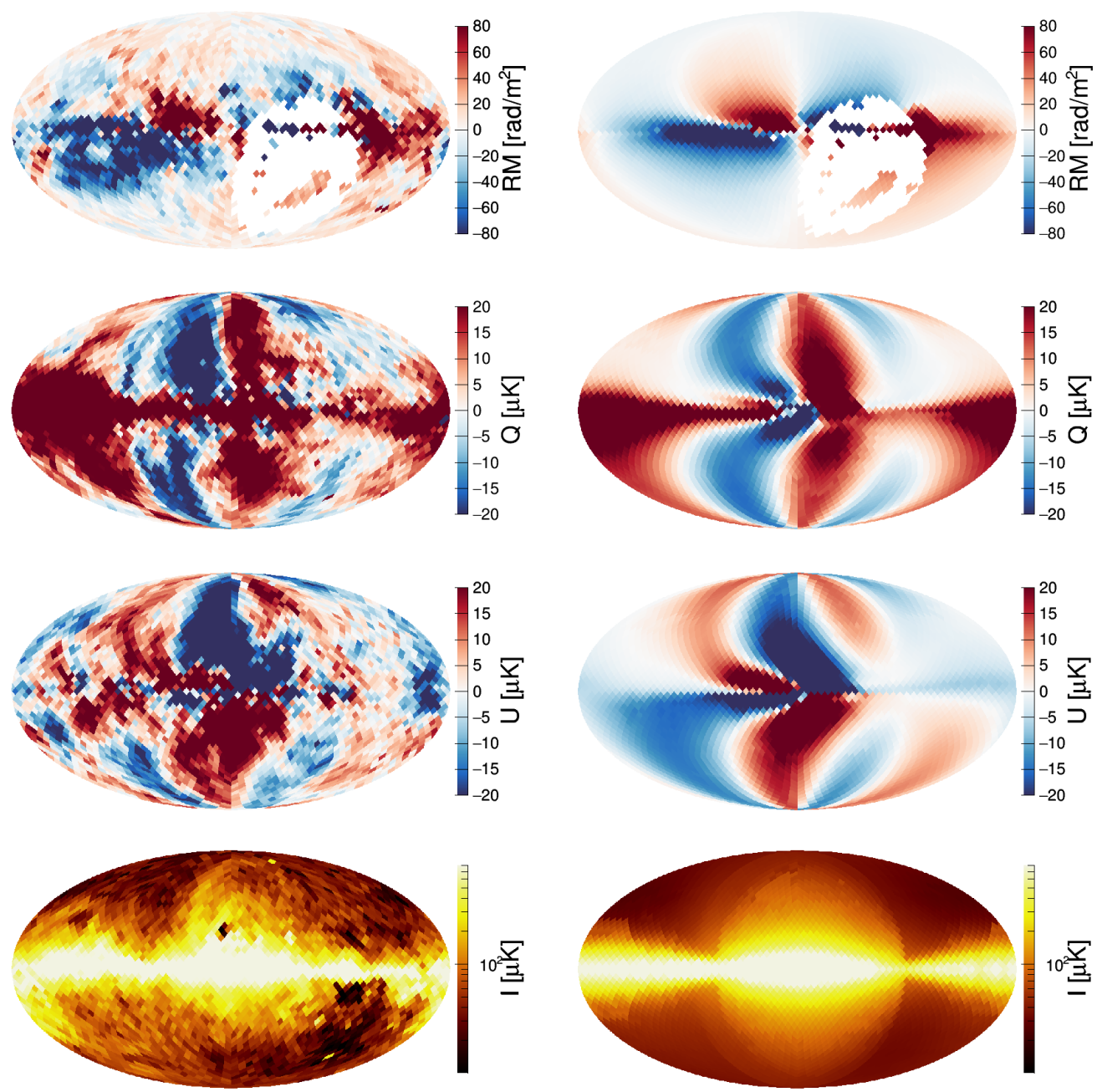

Figure 1: Measured (left column) and simulated (right column) sky maps of rotation measures (top), polarized synchrotron emission (Stokes Q and U parameters in the two middle panels) and total synchrotron intensity (bottom). The synchrotron data are from [6] and the GMF model is JF12 [4, 5].

\section{Data Analysis}

The parameters of the different GMF models are optimized by minimizing the varianceweighted pixel-by-pixel difference between the predicted and measured observables. In [4], this optimization was performed with a time-intensive MCMC sampling of the posterior distribution of the model parameters. The model variations investigated in the present work usually lead to much larger differences in the estimates of the GMF than the single-model statistical uncertainties. 
Therefore the posterior distribution of the parameters are of secondary importance and the $n$ model parameters can be optimized more efficiently with multi-dimensional gradient search [7]. For each model variation, the gradient search is perform typically 50 times in parallel starting at random positions in the $n$-dimensional parameter space to assure that the global minimum is found.

\section{Model Variations}

In the following we will give a brief overview of the investigated modeling variations. The different models are listed in Table 1. As can be seen in the last column of this table, all of the 20 modeling variations give a reasonable description of the data with reduced $\chi^{2}$ s ranging from 0.78 to 1.22 . Note that for all models we use the same relative weighting of the $\chi^{2}$ contributions of the RM and synchrotron data. Moreover, the same pixel mask is applied in all cases (see [4] for a description of the mask). It should be emphasized that due to differences in the data processing (especially in the synchrotron data products), the reduced $\chi^{2}$ is not always appropriate for comparing the fit quality in the model variations, as discussed below.

Parameterizations We tested several alternatives to the parameterizations of the coherent field used in the JF12 model. All these alternatives are divergence-free, like the original functional forms. First, we replaced the parametric X-field of JF12 with "model C" from [8] (variation $b$ ). The main difference between these two models is that in the former the angle of the poloidal field lines with respect to the Galactic plane is kept constant above a certain galacto-centric radius, whereas in "model C" this angle evolves with radius and height. (The smoother behavior of "model C" at the Galactic plane is unobservable due to random fields.) In the next step we restrict the radial extent of the toroidal field to be the same in the Northern and Southern hemisphere of the Galaxy leading to a best-fit value of $r_{\mathrm{N} / \mathrm{S}}=11.3 \pm 0.3 \mathrm{kpc}$ (variation $c$ ). Introducing a Galactic warp [9] into the model (variation $d$ ) leaves most of the line-of-sights unchanged and introduces only minor changes to the simulated observables at low latitudes close to the Galactic anti-center. The disk field of JF12 is implemented as a logarithmic spiral with a pitch angle of $11.5^{\circ}$ and eight discontinuous magnetic field "arms" following [10]. We replaced this ansatz by a divergence-free smooth spiral field with free pitch angle (variation $e$ ). Interestingly, the best fit gives a pitch angle of $(14.5 \pm 0.6)^{\circ}$, i.e. close to the pitch angles determined for the spiral segments of the Milky Way (see e.g. [11]) in accordance to observations of external galaxies [12]. Finally, we replaced the independent toroidal and poloidal components of JF12 by a "twisted X-field", i.e. a originally poloidal field that is evolved in time taking into account the radial and vertical shear of the Galaxy. Allowing for different parametric forms of the radial dependence of the initial field yields model variations $f$ and $g$ [13].

Despite the considerable differences between these parameterization, the quality of the fits is similar for each of them (cf. Table 1), i.e. the data does not clearly discriminate between these model variations at this stage.

Synchrotron Data Products As the baseline for the comparisons considered in this work, we use the 7-year WMAP synchrotron maps [6], as originally used in JF12. But for variations $h-l$ we replace that synchrotron data with different synchrotron products from the 9-year final WMAP data release [14] and the Planck 2015 data release [15]. These products differ in the constraints applied to the measured Galactic microwave emission data to extract the synchrotron component. 


\begin{tabular}{|c|c|c|c|c|c|c|c|c|}
\hline id & $\begin{array}{c}\text { disk } \\
\text { model }\end{array}$ & $\begin{array}{c}\text { toroidal } \\
\text { model }\end{array}$ & $\begin{array}{c}\text { poloidal } \\
\text { model }\end{array}$ & $\begin{array}{l}\text { thermal } \\
\text { electrons }\end{array}$ & $\begin{array}{l}\text { cosmic-ray } \\
\text { electrons }\end{array}$ & $\begin{array}{l}\text { synchrotron } \\
\text { data product }\end{array}$ & misc. & $\chi^{2} / \mathrm{ndf}$ \\
\hline \multicolumn{9}{|c|}{ Parametric models } \\
\hline $\mathrm{a}$ & JF & JF & $\mathrm{JF}$ & NE2001 & $\mathrm{GP}_{\mathrm{JF}}$ & WMAP7 & - & 1.10 \\
\hline b & JF & $\mathrm{JF}$ & FTC & NE2001 & $\mathrm{GP}_{\mathrm{JF}}$ & WMAP7 & - & 1.09 \\
\hline $\mathrm{c}$ & $\mathrm{JF}$ & JFsym & FTC & NE2001 & $\mathrm{GP}_{\mathrm{JF}}$ & WMAP7 & - & 1.11 \\
\hline d & $\mathrm{JF}$ & JFsym & FTC & NE2001 & $\mathrm{GP}_{\mathrm{JF}}$ & WMAP7 & warp & 1.11 \\
\hline $\mathrm{e}$ & UF & JFsym & FTC & NE2001 & $\mathrm{GP}_{\mathrm{JF}}$ & WMAP7 & - & 1.09 \\
\hline $\mathrm{f}$ & UF & \multicolumn{2}{|c|}{$\mathrm{UFa}$} & NE2001 & $\mathrm{GP}_{\mathrm{JF}}$ & WMAP7 & - & 1.14 \\
\hline $\mathrm{g}$ & UF & \multicolumn{2}{|c|}{$\mathrm{UFb}$} & NE2001 & $\mathrm{GP}_{\mathrm{JF}}$ & WMAP7 & - & 1.09 \\
\hline \multicolumn{9}{|c|}{ Synchrotron products } \\
\hline $\mathrm{h}$ & JF & JFsym & FTC & NE2001 & $\mathrm{GP}_{\mathrm{JF}}$ & WMAP9base & - & $1.22^{\dagger}$ \\
\hline $\mathrm{i}$ & JF & JFsym & FTC & NE2001 & $\mathrm{GP}_{\mathrm{JF}}$ & WMAP9sdc & - & $1.24^{\dagger}$ \\
\hline $\mathrm{j}$ & JF & JFsym & FTC & NE2001 & $\mathrm{GP}_{\mathrm{JF}}$ & WMAP9fs & - & $1.11^{\dagger}$ \\
\hline $\mathrm{k}$ & $\mathrm{JF}$ & JFsym & FTC & NE2001 & $\mathrm{GP}_{\mathrm{JF}}$ & WMAP9fss & - & $1.22^{\dagger}$ \\
\hline 1 & JF & JFsym & FTC & NE2001 & $\mathrm{GP}_{\mathrm{JF}}$ & Planck15 & - & $0.78^{\dagger}$ \\
\hline \multicolumn{9}{|c|}{ Thermal electrons } \\
\hline $\mathrm{m}$ & $\mathrm{JF}$ & JFsym & FTC & YMW17 & $\mathrm{GP}_{\mathrm{JF}}$ & WMAP7 & - & 1.21 \\
\hline $\mathrm{n}$ & UF & JFsym & FTC & YMW17 & $\mathrm{GP}_{\mathrm{JF}}$ & WMAP7 & - & 1.14 \\
\hline o & JF & $\mathrm{JF}$ & FTC & NE2001 & $\mathrm{GP}_{\mathrm{JF}}$ & WMAP7 & $\kappa=-1$ & $1.05^{*}$ \\
\hline $\mathrm{p}$ & JF & $\mathrm{JF}$ & FTC & NE2001 & $\mathrm{GP}_{\mathrm{JF}}$ & WMAP7 & $\kappa=+1$ & $1.05^{*}$ \\
\hline$q$ & $\mathrm{JF}$ & JFsym & FTC & NE2001 & $\mathrm{GP}_{\mathrm{JF}}$ & WMAP7 & HIM & 1.12 \\
\hline \multicolumn{9}{|c|}{ Cosmic-ray electrons } \\
\hline $\mathrm{r}$ & JF & JFsym & FTC & NE2001 & O13a & WMAP7 & - & 1.13 \\
\hline s & $\mathrm{JF}$ & JFsym & FTC & NE2001 & $\mathrm{O} 13 \mathrm{~b}$ & WMAP7 & - & 1.12 \\
\hline $\mathrm{t}$ & $\mathrm{JF}$ & JFsym & FTC & NE2001 & $\mathrm{S} 10$ & WMAP7 & - & 1.13 \\
\hline
\end{tabular}

Table 1: Summary of model variations investigated in this paper. The original JF12 model corresponds to the first row (model a) and the reference model is given in the third row (model 3). The goodness of fit for describing the RM, Q and $\mathrm{U}$ data is given in the last column with the exception for the combined fits of coherent and random field (marked with a ${ }^{*}$ ), where the $\chi^{2}$ also includes the contribution from the total intensity I. The $\chi^{2} \mathrm{~s}$ of the fits with different synchrotron data products (marked with $\mathrm{a}^{\dagger}$ ) used different weights in the fits derived from these products.

WMAP7 and WMAP9base fit the data with a sum of synchrotron, free-free and dust emission. All other models include a spinning dust component to describe the "anomalous microwave emission". Moreover, different constraints to the spectral index of the synchrotron emission are applied in different products. It must be emphasized that the sizable differences in the reduced $\chi^{2}$ s for variations $h-l$ visible in Table 1 originate from the different variances inferred from the sub-pixel variation in the data products, reflecting the smoothing procedure of [15]. Thus the relative reduced $\chi^{2}$ s here are not indicative of a better or worse description of the data.

The differences between the various synchrotron products are small for the polarized emission and therefore the inferred coherent magnetic field does not change much when switching from one data product to another. However, as already noted in [16], the different treatment of the anomalous microwave emission in the models used for the different synchrotron products strongly affects the estimated total synchrotron intensities, and thus the random magnetic field component. This difference leads to a reduction of random field strength, by up to a factor of four in the disk, relative to JF12 [13].

Thermal Electrons A model of the density of thermal electrons in the Galaxy $\left(n_{e}\right)$ is needed to predict the rotation measures for a given magnetic field configuration. Estimates of the spatial distribution of $n_{e}$ rely mostly on measurements of the dispersion measures of Galactic pulsars 
and to a lesser extent on scattering measures of Galactic and extragalactic sources. We tested the impact of two different models for the thermal electron densities: NE2001 [17], with the updated scale height of the thick disk from [18], and YMW17 [19]. While the newer YMW17 model relies on more dispersion measures from pulsars with measured distances, the more important difference between the two models lies in their particular parametric choices for the model components, such as the thickness and pitch angles of the spiral arms. Both models give a similar performance when comparing the predicted to measured dispersion measures. The slightly worse $\chi^{2}$ values of the YMW17 variations $m$ and $n$, with respect to the corresponding NE2001 fits $c$ and $e$, can be partially attributed to worse description of the RMs in the direction of the Gum nebula when using YMW17. The main difference in the inferred magnetic fields is a larger field strength in the halo: due to the lower density of electrons at large Galactic height in the YMW17 model, the fitted field strengths for the toroidal and poloidal components are about a factor two larger than if determined using NE2001. We also tested the effect of adding the large-scale hot ionized medium from [20] to the calculation of RMs, but no big effect on the inferred GMF was found (variation $q$ ).

In a further $n_{e}$-related variation, we tested the impact of assuming a correlation, $\kappa$, between the magnetic field strength and the thermal electron densities. As argued in [21], pressure balance in the magneto-ionic medium can lead to an anti-correlation of $n_{e}$ and the magnetic field, leading to an underestimate of the coherent magnetic field strength inferred from rotation measures if the anti-correlation is ignored. On the other hand, compression of the magneto-ionic medium causes an enhancement of both the magnetic field strength and gas densities, leading to positive correlation between $n_{e}$ and the magnetic field resulting in an overestimate of $B_{\text {coh }}$ inferred from RMs with $\kappa=0$. We studied the two extreme cases $\kappa=-1$ (variation $o$ ) and $\kappa=+1$ (variation $p$ ) by performing a combined fit of the coherent and random fields using the modified relations between RM and the magnetic field given in [21]. As expected, very different coherent magnetic field strengths were found for the two cases. The total energy of the coherent field in the Galaxy is $3.5 \times 10^{55} \mathrm{erg}$ for $\kappa=-1$ and $3.8 \times 10^{54} \mathrm{erg}$ for $\kappa=+1$. Under the standard assumption of no correlation (variation $b$ ), the coherent energy is $1.2 \times 10^{55} \mathrm{erg}$. Note that these are upper bounds on the effects of a possible $n_{e}-B$ correlation, because a) the assumed correlation coefficients are at the extreme values and $b$ ) the synchrotron product used in the comparison is the baseline one from WMAP7 without a spinning dust component, whose inferred random magnetic field is largest (cf. previous section).

Cosmic-Ray Electrons The density and spectrum of cosmic-ray electrons depends in two ways on the Galactic magnetic field: Firstly, the GMF determines the diffusion of the electrons from their sources through the Galaxy and, secondly, synchrotron losses at high electron energies is the main cause of electron cooling. The JF12 fits used $n_{\text {cre }}$ from a two-dimensional GALPROP simulation with a uniform isotropic diffusion coefficient within a cylindrical volume of $4 \mathrm{kpc}$ height. Here we tested the three variants of the cosmic-ray electron densities used in [16], which are updated versions of the calculations described in [22] and [23]. Two of them (variation $r$ and $t$ ) use a vertical extent of the diffusion volume of $4 \mathrm{kpc}$, whereas for variation $s$ the height of the diffusion volume is $10 \mathrm{kpc}$. The inferred field strengths of the coherent GMF are remarkably robust under these changes, partially being due to the flexibility in the fit to adjust the relative scale between the RMs and the polarized intensity by changing the amount of striated fields, i.e. aligned anisotropic 


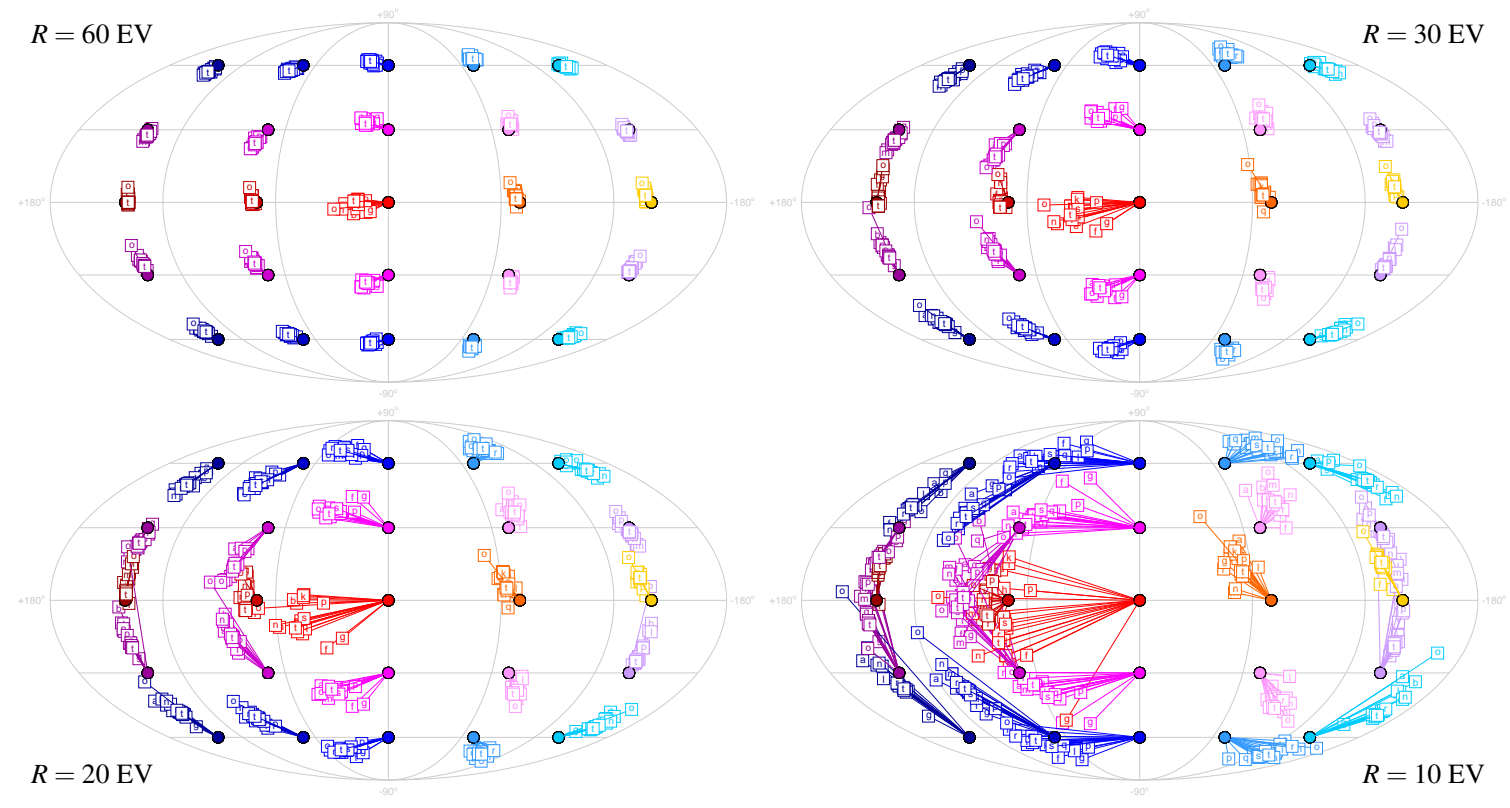

Figure 2: Backtracking of charged particles at different rigidities from a regular grid of initial directions (dots) through different models of the coherent GMF. The resulting directions outside of the Galaxy are denoted by squares and the letters correspond to the models listed in Table 1. The sky maps are in Galactic coordinates and the particle rigidities indicated in corners of each panel.

random fields that contribute to the polarized intensity, but not to the rotation measures. Further studies concerning the impact of cosmic-ray electrons will be shown in [13], in particular the effect of using a more detailed three-dimensional source distribution of relativistic electrons.

\section{Application to Charged Particle Astronomy}

Taken together, the variations of model assumptions explained in the last section lead to an ensemble of models for the Galactic magnetic field, each of which is compatible with the current data on the rotation measures from extragalactic radio sources and the synchrotron emission in the microwave band. This ensemble of models can be considered as a provisional estimation of the uncertainty of our knowledge of the GMF, and used to propagate the uncertainty in our knowledge of the GMF to any kind of calculation involving the magnetic field of the Galaxy, by repeating the calculation for each of the models. The variations of the results give a lower limit on the propagated uncertainty, in the absence of further input to select among or discard some of the GMF model variations.

As an example we present here the uncertainties in the arrival direction of ultrahigh energy cosmic rays, induced by uncertainties of the coherent component of the GMF. (See [24] for a discussion concerning the impact random component of the GMF on deflections and [25] for a comparison of deflections using two models of the coherent GMF.) For this purpose we performed a backtracking of charged particles through each of the GMF models listed in Table 1. The results are shown in Fig. 2 for particle rigidities $R$ (rigidity $=$ energy/charge) of 10, 20, 30 and $60 \mathrm{EV}$. As can be seen, for very large rigidities (e.g. protons with energies of $6 \times 10^{19} \mathrm{eV}$ ), the overall 
amount of deflection and correspondingly also the model differences are small, confirming the long-speculated possibility of charged particle astronomy with protons at ultrahigh energies. Even for rigidities as low as $20 \mathrm{EV}$, the different deflections are mostly confined within well-defined regions so it seems plausible that a correction for the spatially varying average deflection based on all models, can still be used to enhance the capabilities to identify the sources of ultrahigh energy cosmic rays. For still lower rigidities, e.g. $10 \mathrm{EV}$, the differences in the backtracked directions start to diverge considerably, but even in this case, a more sophisticated analysis can reduce the current uncertainties in studies of the source direction of ultrahigh energy cosmic ray nuclei. Further progress on constraining the magnetic field is also expected.

\section{Acknowledgments}

We would like to thank Tess Jaffe for providing the simulations for the cosmic-ray electrons models from [16]. MU acknowledges the financial support from the EU-funded Marie Curie Outgoing Fellowship, Grant PIOF-GA-2013-624803 and would like to thank CCPP/NYU for their hospitality. The research of GRF is supported in part by the U.S. National Science Foundation (NSF), Grant NSF-1517319.

\section{References}

[1] A. Shukurov, "Introduction to galactic dynamos," in Mathematical Aspects of Natural Dynamos, pp. 319-366. 2004.

[2] M. Haverkorn, "Magnetic Fields in the Milky Way," in Magnetic Fields in Diffuse Media, vol. 407 of Astrophysics and Space Science Library, p. 483. 2015.

[3] R. Beck A\&A Rev. 24 (2016) 4.

[4] R. Jansson and G. R. Farrar ApJ 757 (2012) 14.

[5] R. Jansson and G. R. Farrar ApJ 761 (2012) L11.

[6] B. Gold et al., [WMAP Collab.] ApJS 192 (2011) 15.

[7] F. James and M. Roos Computer Physics Communications 10 (1975) 343-367.

[8] K. Ferrière and P. Terral A\&A 561 (2014) A100.

[9] E. S. Levine, L. Blitz, and C. Heiles ApJ 643 (2006) 881-896.

[10] J. C. Brown et al. ApJ 663 (2007) 258-266.

[11] M. J. Reid et al. ApJ 783 (2014) 130.

[12] L. Chamandy, A. Shukurov, and A. R. Taylor ApJ 833 (2016) 43.
[13] M. Unger and G. R. Farrar in preparation (2017) .

[14] C. L. Bennett et al., [WMAP Collab.] ApJS 208 (2013) 20.

[15] R. Adam et al., [Planck Collab.] A\&A 594 (2016) A10.

[16] R. Adam et al., [Planck Collab.] A\&A 596 (2016) A103.

[17] J. M. Cordes and T. J. W. Lazio arXiv:0207156 [astro-ph] (2002) .

[18] B. M. Gaensler, G. J. Madsen, S. Chatterjee, and S. A. Mao PASA 25 (2008) 184-200.

[19] J. M. Yao, R. N. Manchester, and N. Wang ApJ 835 (2017) 29.

[20] M. J. Miller and J. N. Bregman ApJ 800 (2015) 14.

[21] R. Beck, A. Shukurov, D. Sokoloff, and R. Wielebinski A\&A 411 (2003) 99-107.

[22] A. W. Strong et al. ApJ 722 (2010) L58-L63.

[23] E. Orlando and A. Strong MNRAS 436 (2013) 2127-2142.

[24] G. R. Farrar, N. Awal, D. Khurana, and M. Sutherland Proc. 34th ICRC (2015) .

[25] M. Erdmann, G. Müller, M. Urban, and M. Wirtz Astropart. Phys. 85 (2016) 54-64. 